\title{
Dramatic Reduction of CEA Post Spray Cryotherapy in a Patient with Pleural Based Malignancy
}

\section{Gordon Downie ${ }^{1}$, Michael Lewis ${ }^{2}$ and Adam Wellikoff ${ }^{3 *}$}

${ }^{1}$ Division of Interventional Pulmonology, Titus Regional Medical Center, USA

${ }^{2}$ Division of Thoracic Surgery, Titus Regional Medical Center, USA

${ }^{3}$ Division of Pulmonary and Critical Care Medicine, LSU Health Shreveport, USA

\begin{abstract}
Malignancy of the parietal pleural is a therapeutic challenge; current therapies largely target patient's regional symptoms related to effusions/tumor burden and restrictive physiology. Seldom are tumor ablation procedures considered. If alternatives to the current standard approaches could promote tumor ablation, address symptoms and be cost effective, studies to assess clinical impact of these alternatives may be warranted. We present a novel cryobased technology for treating pleural malignant disease with a dramatic reduction in a tumor biomarker.
\end{abstract}

We present a case of a 67-year-old man with mucinous adenocarcinoma of the pleura with elevated Carcinoembryonic Antigen (CEA) level who had an inadequate response to standard treatment. He underwent cryospray ablation of the parietal pleura with tumor destruction being documented both visually and with a significant decrease in his CEA level.

This case demonstrates tumor necrosis, a rapid drop in serum CEA, as well as ease of treatment and continued evidence of safety and the feasibility of cryospray ablation in the pleural space. This case demonstrated that spray cryotherapy promoted tumor ablation and addressed symptoms within the pleura thereby indicating the need for further study. The degree of tumor necrosis in multiple clinical presentations (bulky, flat, studding, etc) awaits definition, as does the rate and timing of post-treatment pleurodesis and the possibility of an immunoregulatory or mortality effect.

Keywords: Cryotherapy; Pleura; Malignancy; Lung cancer; CEA

\section{Introduction}

Malignancy of the parietal pleura is a therapeutic challenge; current therapies largely target patient's regional symptoms that can be related to large effusions/tumor burden and restrictive physiology. Seldom is tumor burden impact or methods that would allow for tumor ablation considered. Lack of consideration is mainly due to concerns of efficacy and cost. If alternatives to the current standard approaches could promote tumor ablation, address symptoms and be cost effective, studies to assess clinical impact of these alternatives may be warranted. We present a novel cryo-based technology for treating pleural malignant disease with a dramatic reduction of a patient's measured Carcinoembryonic Antigen (CEA) as direct evidence of tumor ablation.

Cancer within the thorax is the most frequently diagnosed non-skin cancer in both men and women, and the leading cause of cancer death in the United States. The National Cancer Institute estimated that more than 230,000 Americans would be diagnosed with lung cancer in 2007 [1]. Pleural-based cancers are not specifically recorded in the American Cancer Society's 2007 new diagnosis estimates. The historical 5-year survival rate for all lung cancer is approximately $14 \%$. The 5 -year survival rate for pleural-based cancer is even lower, but it is unknown what clinical impact ablation of pleural-based metastasis will have given the dogma that pleural based disease is a marker of systemic spread. Current standard of care treatment for pleural metastasis is targeted at symptom attenuation via pleurodesis associated with pleural decortication, in some case. A less invasive, more tolerable method of reducing symptoms, accompanied by a reduction in tumor burden, would represent a substantive improvement relative to current approaches.

Treatment for pleural malignancy over the last 20 years has included regional application of chemotherapeutics, phototherapy, cyrotherapies, radiation and immunotherapies with limited success. Specifically cryo-based applications have included post-thoracotomy pain reduction, biopsies, ablation and combination therapies including chemotherapy with cryotherapy [2-5].

Standard, slow-energy transfer cryotherapy or cryosurgery has been used in the thorax for many decades, and there is an excellent understanding of the biologic principles governing these slow-energy transfer devices as well as the cryobiologic response in those applications [6-11]. Slow-energy transfer devices utilize the Joule-Thompson effect comprising the expansion of gas resulting in thermal cooling. Among the limitations with this approach has been a narrow field of effect, obligatory mechanical contact with the target, and inconsistent depth of injury as thermal transfer rates are limited by lowest attainable temperature - typically -85 degrees centigrade. Spray cryotherapy using low-pressure (less than 4 PSI) delivery of liquid nitrogen at nearly -200 degrees centigrade addresses many of these deficiencies. It is a rapid energy transfer platform using liquid nitrogen with significantly more efficient energy transfer. This FDA-certified, low-pressure, spray cryotherapy device may be used to destroy unwanted tissues and was initially introduced in the GI tract and with thousands of procedures completed to date has proven to be not only safe, but reproducible and with durable results as well [6]. We published our first case of use within the pleural space and reported observations on this technology's safety and feasibility in that setting [6]. In the current case, we report sustained safety and feasibility as well as a demonstration of clinical efficacy by both directly observed tissue necrosis and a significant reduction in our patient's serum CEA.

\section{Case Report}

A 67-year-old male, who is a practicing medical oncologist, was

*Corresponding author: Adam Wellikoff, MD, LSU Health, Section of Pulmonary, Critical Care and Sleep Medicine, 1501 Kings Hwy, Shreveport, LA 71130, USA, Tel: 318-675-5920; Fax: 318-675-5988; E-mail: awelli@Isuhsc.edu

Received April 23, 2013; Accepted June 14, 2013; Published June 17, 2013

Citation: Downie G, Lewis M, Wellikoff A (2013) Dramatic Reduction of CEA Pos Spray Cryotherapy in a Patient with Pleural Based Malignancy. J Pulmon Resp Med S14: 005. doi:10.4172/2161-105X.S14-005

Copyright: $\odot 2013$ Downie G, et al. This is an open-access article distributed under the terms of the Creative Commons Attribution License, which permits unrestricted use, distribution, and reproduction in any medium, provided the original author and source are credited. 
being followed locally for a slowly rising CEA level. The CEA increased from $5 \mathrm{ng} / \mathrm{mL}$ to $20 \mathrm{ng} / \mathrm{mL}$ over a five-year period. The patient underwent colonoscopy twice along with imaging including chest $\mathrm{x}$-ray, Computed Tomography (CT) scans, and a Positron Emission Tomography (PET) scan all of which were negative. Over the next four years the CEA increased to $40 \mathrm{ng} / \mathrm{mL}$ and a follow up PET/CT showed increased fluorine-18 (F-18) Fluorodeoxyglucose (FDG) uptake in the left pleura. He underwent Video-Assisted Thoracoscopic Surgery (VATS) one month later, which conformed low-grade mucinous adenocarcinoma of the pleura. His CEA at the time of the procedure was $63.7 \mathrm{ng} / \mathrm{mL}$. Immunohistochemical stains ruled out mesothelioma and molecular testing showed that Epidermal Growth Factor Receptor (EGFR), V-Ki-ras2 Kirsten Rat Sarcoma viral oncogene homolog (KRAS), and echinoderm microtubule-associated protein-like 4 geneanaplastic lymphoma kinase (EML4-ALK) were negative.

He underwent treatment with erlotinib and bevacizumab that had to be stopped 5 months into treatment because of toxicity. He was then started on carboplatin, pemetrexed, and bevacizumab for three cycles. The CEA decreased to $29.3 \mathrm{ng} / \mathrm{mL}$ after his initial chemotherapy treatment but increased afterwards to $124 \mathrm{ng} / \mathrm{mL}$ with no response to the second-line treatment. The patient then presented for consideration of cryospray ablation versus pleurectomy followed by photodynamic therapy. The patient is a high-level amateur athlete and performance status and rapid recovery from the intervention were priorities. $\mathrm{He}$ therefore chose spray cryotherapy and underwent a VATS procedure with treatment applied to the left parietal pleura. Upon entry to the pleural space it was noted that disease was present diffusely on the parietal pleura although some areas had more bulky disease than others. Eleven of the most densely involved locations were selected for therapy which encompassed the majority of the parietal pleura. Each site was treated with five cycles of ten-second sprays with a sixty-second thaw cycle. Once the treated area thawed, each site showed varying degrees of cherry-red hyperemia thought to represent necrosis. No surrounding vital structures were treated as the application of the cryospray can be delivered to very specific locations with ease. The patient tolerated the procedure well with no complications and was discharged two days later. Six days after the cryospray procedure the CEA decreased to 42.8 $\mathrm{ng} / \mathrm{mL}$, which persisted to one month out.

A follow up CT done one month after the cryospray showed decrease in size of the pleural lesions at $3.6 \mathrm{~mm}$ compared to $8.0 \mathrm{~mm}$ on imaging done 2 months prior. (Figure 1 and 2) At last follow up 5 months after the procedure, the patient's functional status has been stable. His CEA level has increased slightly to approximately $60 \mathrm{ng} / \mathrm{mL}$ but the CT remains stable as has his most recent CEA levels (Structure 1).

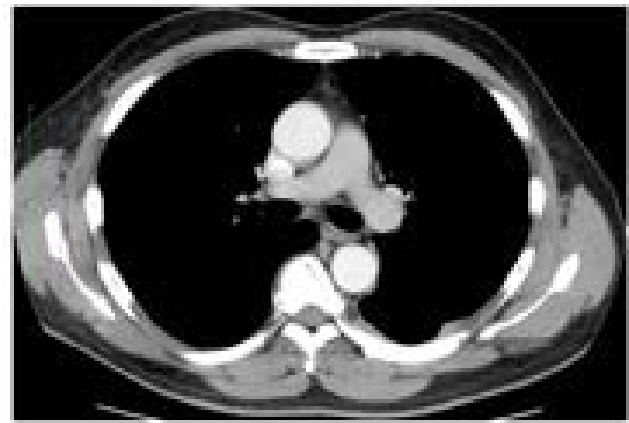

Figure 1: March2011 (pre-cryospray).



Figure 2: September 2011 (post-cryospray)

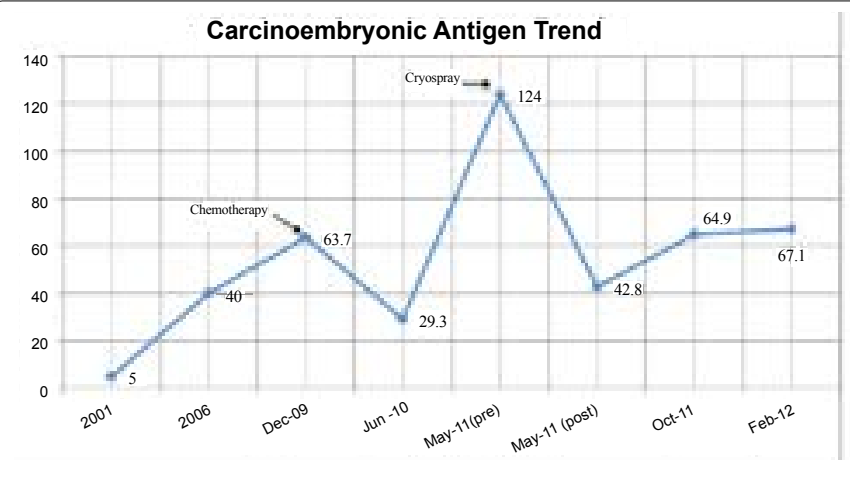

Structure 1.

\section{Discussion}

We present the use of spray cryotherapy in the treatment of a patient with cancer in the pleural space. We observed consistency of the safety and feasibility parameters we previously have seen and reported but additionally demonstrated a rapid decline in this patient's serum CEA that correlated with the extent of initial tumor necrosis observed during the VATS procedure.

Pleurodesis and, when necessary, decortication of the affected pleural space are the current standard of care for Mla non-small cell lung cancer and are certainly effective at addressing dyspnea and effusion related sequelae. This process however is often fairly invasive and typically requires extended hospital stays and convalescence. Barriers for direct tumor treatment options within the pleura are cost, complex dosimetry, and potential futile intervention if survival is unaffected. Novel strategies to mitigate symptoms while at the same time defining and reducing the pleural tumor burden may be of value but gain little traction because of the previously mentioned barriers.

This case affords a significant opportunity to glean the potential of spray cryotherapy in pleural metastases because several usually confounding variables are not present. This patient had no recent oncologic treatment for this slowly progressive adenocarcinoma after his initial first and second-line chemotherapy. Additionally, he had serial measurements of CEA that have been tracked for years, so a rapid decline would likely be related to tumor cell death and not lab variability. The slow doubling time of this cancer, its limited location and the patient's status as a practicing medical oncologist optimized the interview process and gave us high confidence in the accuracy of the responses. Lastly, the VATS approach with a completely collapsed lung and the length of the procedure provided for extended time to observe 
pleural tumor reaction to the treated sites, as well as a very accurate estimate of the percentage of tumor burden present and treated within the left thorax.

We are confident that the observed rapid decline in the patient's serum CEA directly correlates with the percentage of tumor burden treated by spray cryotherapy and that the decline represents tumor cell death directly related to the use of the cryotherapy. To date, animal studies conducted looking at the histologic changes associated with cryospray have shown the extent of cell death are variable depending on the tissue treated. Cell death has been observed $2-4 \mathrm{~cm}$ from the spray area attributed to disruption of the vascular bed rather than direct injury.

Cryobiologic therapies are attractive for the pleural space for several reasons including relative ease of access to the pleural surface, cryoresistant support structures that help attenuate bystander side effects, low complexity dosimetry and competitive costs. The cryogen is delivered in the form of a liquid spray via a nozzle that allows for fine control with an exact treatment edge further reducing the possibility of bystander damage.

Pain is another prominent feature of pleural-based malignancies and although pleuritic pain was not a significant finding in this case there are decades of reported positive outcomes from cryoanalgesia in postthoracotomy patients [6,3]. Additionally, well-documented immune-regulatory effects have been described with cryotherapy, raising the possibility of a systemic immune response from this local therapy [10].

\section{Conclusion}

To summarize, the initial observations from this case include: observed rapid tumor necrosis with a correlated drop in serum CEA, ease of treatment and continued evidence of safety and the feasibility of this technique. This case illustrates the potential of addressing malignant pleural disease with a low-cost, lowcomplexity technology with the potential to affect the parameters that affect survival. Although a single case report is not adequate to apply to a broader population, we believe that spray cryotherapy represents a viable opportunity for further study within the pleura. The degree of tumor necrosis in multiple clinical presentations (bulky, flat, studding, etc) awaits definition, as does the rate and timing of post-treatment pleurodesis and the possibility of an immunoregulatory effect or an impact on mortality.

\section{References}

1. American Cancer Society (2007) Cancer Facts and Figures.

2. Glynn CJ, Lloyd JW, Barnard JD (1980) Cryoanalgesia in the management of pain after thoracotomy. Thorax 35: 325-327.

3. Forest V, Peoc'h M, Ardiet C, Campos L, Guyotat D, et al. (2005) In vivo cryochemotherapy of a human lung cancer model. Cryobiology 51: 92-101.

4. Forest V, Hadjeres R, Bertrand R, Jean-François R (2009) Optimisation and molecular signalling of apoptosis in sequential cryotherapy and chemotherapy combination in human $\mathrm{A} 549$ lung cancer xenografts in SCID mice. Br J Cance 100: 1896-1902.

5. Downie G, Krimsky W (2010) Response to spray cryotherapy in a patient with adenocarcinoma in the parietal pleura. Respiration 80: 73-77

6. Vergnon JM, Huber RM, Moghissi K (2006) Place of cryotherapy, brachytherapy and photodynamic therapy in therapeutic bronchoscopy of lung cancers. Eur Respir J 28: 200-218.

7. Neel HB 3rd, Farrell KH, DeSanto LW, Payne WS, Sanderson DR (1973) Cryosurgery of respiratory structures. I. Cryonecrosis of trachea and bronchus. Laryngoscope 83: 1062-1071.

8. Rubinsky B (2000) Cryosurgery. Annu Rev Biomed Eng 2: 157-187.

9. Baust JG, Gage AA (2004) Progress toward optimization of cryosurgery. Technol Cancer Res Treat 3: 95-101.

10. Sabel MS (2009) Cryo-immunology: a review of the literature and proposed mechanisms for stimulatory versus suppressive immune responses. Cryobiology 58: 1-11.

11. Krimsky W, Sarkar S, McKenzie K (2008) Safety and efficacy of cryospray ablation in the airway of healthy swine Am J Respir Crit Care Med 177: A893.
This article was originally published in a special issue, Pulmonology- Case Reportshandled by Editor(s). Dr. Anna Blumental-Perry, Memorial University Medical Center, USA. Dr. Bradley Allen Maron, Brigham and Women's Hospital, USA. 